\title{
Acoustic cues to individuality in wild male adult African savannah elephants (Loxodonta africana)
}

\author{
Kaja Wierucka ${ }^{\text {Corresp., }}{ }^{,}$Michelle D Henley ${ }^{2,3}$, Hannah S Mumby ${ }^{1,4,5}$ \\ ${ }^{1}$ School of Biological Sciences, University of Hong Kong, Hong Kong, Hong Kong \\ 2 Applied Ecosystem and Conservation Research Unit, University of South Africa, Johannesburg, South Africa \\ 3 Elephants Alive, Hoedspruit, South Africa \\ 4 Department of Zoology, University of Cambridge, Cambridge, United Kingdom \\ ${ }^{5}$ Centre for African Ecology, School of Animal, Plant and Environmental Sciences, University of Witwatersrand, Wits, South Africa \\ Corresponding Author: Kaja Wierucka \\ Email address: wierucka@hku.hk
}

The ability to recognize conspecifics plays a pivotal role in animal communication systems. It is especially important for establishing and maintaining associations among individuals of social, long-lived species, such as elephants. While research on female elephant sociality and communication is prevalent, until recently male elephants have been considered far less social than females. This resulted in a dearth of information about their communication and recognition abilities. With new knowledge about the intricacies of the male elephant social structure come questions regarding the communication basis that allows for social bonds to be established and maintained. By analyzing the acoustic parameters of social rumbles recorded over 1.5 years from wild, mature, male African savanna elephants (Loxodonta africana) we expand current knowledge about the information encoded within these vocalizations and their potential to facilitate individual recognition. We showed that social rumbles are individually distinct and stable over time and therefore provide an acoustic basis for individual recognition. Furthermore, our results revealed that different frequency parameters contribute to individual differences of these vocalizations. 


\title{
1 Acoustic cues to individuality in wild male adult
}

\section{African savannah elephants (Loxodonta africana)}

3

\author{
Kaja Wierucka ${ }^{1}$, Michelle D. Henley ${ }^{2,3}$, Hannah S. Mumby ${ }^{1,4,5}$ \\ ${ }^{1}$ School of Biological Sciences, University of Hong Kong, Hong Kong \\ 2 Applied Ecosystem and Conservation Research Unit, University of South Africa, \\ Johannesburg, South Africa \\ ${ }^{3}$ Elephants Alive, Hoedspruit, South Africa \\ ${ }^{4}$ Department of Zoology, University of Cambridge, Cambridge, United Kingdom \\ ${ }^{5}$ Centre for African Ecology, School of Animal, Plant and Environmental Sciences, University \\ of Witwatersrand, Wits, South Africa
}

\section{Corresponding Author:}

Kaja Wierucka

School of Biological Sciences, Kadoorie Biological Sciences Building, The University of Hong Kong, Pok Fu Lam Road, Hong Kong

Email address: wierucka@hku.hk

\section{Abstract}

The ability to recognize conspecifics plays a pivotal role in animal communication systems. It is especially important for establishing and maintaining associations among individuals of social, long-lived species, such as elephants. While research on female elephant sociality and communication is prevalent, until recently male elephants have been considered far less social than females. This resulted in a dearth of information about their communication and recognition abilities. With new knowledge about the intricacies of the male elephant social structure come questions regarding the communication basis that allows for social bonds to be established and maintained. By analyzing the acoustic parameters of social rumbles recorded over 1.5 years from wild, mature, male African savanna elephants (Loxodonta africana) we expand current knowledge about the information encoded within these vocalizations and their potential to facilitate individual recognition. We showed that social rumbles are individually distinct and stable over time and therefore provide an acoustic basis for individual recognition. Furthermore, our results revealed that different frequency parameters contribute to individual differences of these vocalizations. 


\section{Introduction}

41 Communication plays an important role in social interactions among animals (Enquist et al.

42

43

44

45

46

47

48

49

50

51

52

53

54

55

56

57

58

59

60

61

62

63

64

65

66

67

68

69

70

71

72

73

74

75

76

77

78
2010). It is an essential component of a wide variety of behaviors related to mating, parental care, predator-prey interactions, group cohesion, and foraging (Bradbury and Vehrencamp 2011). However, for many of these interactions to take place, animals must possess the ability to recognize others. Recognition can vary in specificity, from discrimination of a species, to recognition of sex, kin, mates, rivals or even specific individuals (Tibbetts and Dale 2007). It is particularly important when repeated interactions occur within a group of conspecifics as it allows individuals to adjust their behavioral response based on previous encounters (Yorzinski 2017). Individual recognition is one the most complex forms of recognition and takes place when individually distinctive characteristics encoded within signals or cues are used by animals for the identification of others (Tibbetts and Dale 2007, Carlson et al. 2020).

Recognition can be achieved through many sensory modalities, yet different cues are subject to limitations resulting from the physical properties of cues and the anatomical features of the animal, and these limitations determine which sensory modality is most effective in a given context (Bradbury and Vehrencamp 2011, Higham and Hebets 2013). Here, we focus on acoustic cues. Acoustic cues are used by many social species to regulate various behavioral processes, including recognition (Owings and Morton 1998; Tibbetts and Dale 2007). They usually communicate immediate states as they do not persist in the environment (Bradbury and Verencamp 2011). However, they can be used over long distances, with low frequency sound propagating further and not subject to scattering to the extent of high frequency sound (Bradbury and Verencamp 2011). Consequently, utilizing acoustic cues for individual discrimination is beneficial when there is a need to broadcast or perceive identity information at a distance. For example, when approaching other individuals is costly (e.g. Falls 1982; Wierucka et al. 2018a, b); or when the environment limits the use of other cues, such as in water (Caldwell and Caldwell 1965).

African savanna elephants (Loxodonta africana) produce a range of vocalizations, including low frequency calls, called rumbles, that are used in various social contexts (Poole et al. 2011; Morris-Drake and Mumby 2017). Vocal communication and recognition have been extensively studied for this species, with rumbles shown to encode sex (Baotic and Stoeger 2017), age (Stoeger et al. 2014), and reproductive (Soltis et al. 2005b) as well as emotional state (Soltis et al. 2005b, Soltis et al. 2009, Wesolek et al. 2009). African savanna elephants use rumbles for the recognition of familiar (Stoeger and Baotic 2017) and family/bond group members (McComb et al. 2000) and to mediate inter-partner distance (Leighty et al. 2008; Soltis et al. 2005a). The species has been shown to retain long-term memory of conspecifics' calls (McComb et al. 2000) and have been shown to produce individually distinct calls (McComb et al. 2003, Soltis et al. 2005b, Clemins et al. 2005). While African elephant rumbles and the information they convey has been extensively studied, a vast majority of this research focused on females and there is relatively little information about acoustic cues produced by males, with only one study

Peer) reviewing PDF | (2020:08:52158:2:0:NEW 15 Dec 2020) 
79 investigating non-musth vocal communication (Stoeger and Baotic 2016). This is a result of the

80 characteristics of the elephant social structure. African savanna elephants live in stable,

81 matrilineal groups and repeated interactions among females are easily observed, with their social

82 structure and association patterns well explored (as summarized in Moss et al. 2011). As a result,

83 the communication basis that allows for complex social bonds among females to be developed

84 has also been studied in detail.

85 African savanna elephant males disperse from their natal groups (Lee et al. 2011) and mature

86 males have been previously thought to live mostly solitary lives. Studies on male-male

87 interactions have focused primarily on males in musth - a state of heightened sexual activity,

88 during which animals are highly aggressive (Poole 1987; e.g. Hollister-Smith et al. 2008,

89 Ganswindt et al. 2005). However, recent studies have shown that mature males outside of the

90 sexually active period are a lot more social than previously assumed (Chiyo et al. 2011,

91 Goldenberg et al. 2014), with stable, long-term relationships occurring over time (Murphy et al.

92 2019). The centrality of animals within a network does not seem to be affected by the age (and

93 thus size) of the animals (Murphy et al. 2019), meaning that they are likely established on an

94 individual basis. If males interact with each other regularly, the ability to identify conspecifics

95 based on individually distinct acoustic cues, would be beneficial for the maintenance of long-

96 term associations and dominance hierarchy.

97 Previous research has shown that information about individuality can be conveyed in male

98 African elephant rumbles (Stoeger and Baotic 2016). This study provided much needed insight

99 into male vocalizations, yet it was conducted on animals living under human care and over a

100 relatively short period of time. For animals to be able to match the cue to a known cognitive

101 template of an individual, cues must not only be unique to a specific individual and different

102 from that of others, but must be stable over time (or the rate of change in a cue must be less than

103 the frequency of interactions between individuals; Thom and Hurst 2004). It is thus important to

104 show that cues can be matched to an individual over longer periods of time, as it is possible that

105 a shorter study may result in similarity being related to context- or state- dependent factors,

106 rather than a long-term basis for individual recognition. Therefore, in this study, we aimed to

107 expand on earlier research by investigating rumbles produced by wild male African elephants

108 recorded over 1.5 years, to determine whether patterns of individual distinctiveness are stable

109 over time and to confirm their potential to facilitate individual recognition in a natural setting.

110

111 Materials \& Methods

112 Data collection

113 The data were collected between June 2016 and October 2017 in the Associated Private Nature 114 Reserves (APNR) in South Africa $\left(24^{\circ} 18^{\prime} \mathrm{S}, 31^{\circ} 18^{\prime} \mathrm{E}\right)$. The APNR is an area of approximately

$11520,800 \mathrm{~km} 2$, adjacent to Kruger National Park, encompassing multiple privately-owned nature 
116 reserves. Although the western border is fenced, the individual reserves to the east are unfenced,

117 as is the boundary to Kruger National Park, allowing for unrestricted movement of animals.

118 Rumbles of adult male elephants were recorded at a sampling frequency of $44.1 \mathrm{kHz}$ on a

119 Marantz PMD661 MKI recorder connected to an Earthworks QTC50 omnidirectional

120 microphone (with a $3 \mathrm{~Hz}-50 \mathrm{kHz}$ flat frequency response), while the animals were $23.5 \mathrm{~m}$ (mean,

$121 \mathrm{SD}=14.4$ ) away from the microphone. Rumbles are very distinct, low frequency calls that cannot

122 be confused with other types of vocalizations produced by elephants (Soltis et al. 2010).

123 Individual identity of males was established visually during recording sessions by assessing the

124 pattern of ear tears and holes and markers of age and sex, then confirmed based on photo-

125 identification methods (following Black et al. 2019) after returning to field base. The elephants

126 in this study were collared (as a part of a different, ongoing long-term project), allowing us to

127 maximize the number of sightings and rumble recordings. To eliminate the influence of age and

128 sex on acoustic parameters (Stoeger and Baotic 2016) and focus on individual differences, we

129 recorded vocalizations of only mature males (over 35 years of age; age was determined

130 following Black et al. 2019). Furthermore, to test for individual differences in a general social

131 context we focused our efforts only on non-musth males. During musth males produce distinct

132 musth-rumbles encoding their sexual state (Poole 1987) that are quantitatively different from

133 rumbles produced during inter-musth periods (Poole 1999). Therefore, animals that were

134 acoustically sampled did not show typical signs of musth (urine-dribbling, urine staining on back

135 legs, temporal gland secretions or temporal gland swelling; Poole 1987) at the time of recording.

136 All sampled animals inhabit the same area, therefore regional differences were not a relevant

137 factor. As our aim was to evaluate the distinctiveness of rumbles across naturally occurring

138 conditions, we did not attempt to limit the recordings to a specific behavioral or social context.

139 Therefore, elephants were sampled at random, with rumbles recorded from animals exhibiting a

140 variety of behaviors (foraging, resting, socializing, traveling, combination). However, to avoid

141 rumbles that may have been produced in a reproductive context, we limited the data to

142 vocalizations produced by males when no females were within sight.

143 All recordings were collected as part of field surveys by the South African non-profit Elephants

144 Alive in line with their agreements with the management of the Associated Private Nature

145 Reserves. The research forms part of a registered and approved SANParks project, in association

146 with the Kruger National Park and Scientific Services and the Associated Private Nature

147 Reserves (Project ID: judith1547.22).

148 Data processing and statistical analysis

149 Rumbles were processed in Raven Pro 1.5. The spectrogram settings were set to a Hann window 150 size of $600 \mathrm{~ms}$, with a hop size of $300 \mathrm{~ms}$ and an overlap of $50 \%$. We only selected rumbles that 151 were of good quality (clearly visible on the spectrogram, with no overlapping vocalizations).

152 Rumbles were identified manually by selecting an area encompassing the entire rumble on the 
153

154

155

156

157

158

159

160

161

162

163

164

165

166

167

168

169

170

171

172

173

174

175

176

177

178

179

180

181

182

183

184

185

186

187

188

189

190

191

spectrogram (Fig. 1). We focused on parameters describing the frequencies and duration of the acoustic cue as frequency values (including the fundamental frequency) have been previously determined important in encoding individual identity information in African elephants (McComb et al. 2003, Stoeger and Baotic 2016). To keep spectral measurements unbiased and consistent as possible, only robust measurements of each rumble were included in the analysis (Table 1). These measurements consider the energy that is stored in the selection rather than time and frequency endpoints, making them unbiased from observer selection (Charif et al. 2010). We measured the Center Frequency, Frequency 5\%, Frequency 95\%, Bandwidth, and Duration 90\% (Charif et al. 2010; Table 1; Fig. 1). While Frequency 5\% is by definition "the frequency that divides the selection into two frequency intervals containing $5 \%$ and $95 \%$ of the energy in the selection" (Charif et al. 2010), in practice, in the case of elephant rumbles, Frequency 5\% is equivalent to the fundamental frequency.

Following the standardization of each variable to a range of 0-1 (to avoid abundance bias in our results), we used a permutational multivariate analysis of variance (PERMANOVA; Anderson 2001; using the vegan package; Oksanen et al. 2019), incorporating Euclidean distances in the matrix, to test whether differences in frequency parameters exist among individuals. This nonparametric method allows for considering multiple variables at low sample sizes to identify overall differences (across individuals) and is appropriate for unbalanced data. To confirm that observed differences are in fact a result of differences across individuals and not an artefact of large differences in within-individual variability, we conducted an analysis of multivariate homogeneity ('betadisper'; Anderson 2001) combined with an ANOVA. We then performed a pairwise comparison (RVAideMemoire package (Hervé 2019); using a Wilk's test, and false discovery rate method for p-value adjustment) along with a SIMPER analysis (Clarke 1993) to determine which variables contributed most to the observed differences. All statistical analyses were conducted in $\mathrm{R}$ version 4.0.2 ( $\mathrm{R}$ Core Team 2020).

\section{Results}

The final database included 81 rumbles from five identified, mature males, over a long time period (an average of 402.8 days between the first and last recording; Table 2). Rumbles had a mean Duration $90 \%$ of $4.19 \mathrm{~s}(\mathrm{SD}=1.05)$ and mean Center Frequency of $28.37 \mathrm{~Hz}(\mathrm{SD}=6.87$; Table 2). Mean Frequency 5\% for elephant rumbles found in our study was $11.65 \mathrm{~Hz}$, which is consistent with the average fundamental frequency of 9.91-13.81 Hz reported for African elephant male rumbles for a similar maturity group (and therefore similar size; Stoeger et al. 2016), confirming that this measurement is, in practice, equivalent to the fundamental frequency (Figure 1). We found significant individual differences in measured spectral features of wild male social rumbles $(\mathrm{R} 2=0.22, \mathrm{p}=0.0001)$. Results of the multivariate homogeneity analysis were not significant $(\mathrm{F}=1.6, \mathrm{df}=4, \mathrm{p}=0.173)$, indicating that the assumption of homogeneity of variances was met by our data and differences across individuals could not be attributed to differences in within-individual variability. Pairwise comparisons showed that even after the 
192 adjustment of $\mathrm{p}$ values for multiple comparisons, the differences between acoustic characteristics 193 of calls were significant for a majority of pairs of individuals (Table 3). These differences were

194 not centred or clustered around specific individuals (no one individual was significantly different 195 than others; Table 3), but rather reflected a random variation of individual differences. SIMPER 196 analyses indicated that the overall contribution of measured spectral parameters to the observed 197 differences was relatively even, ranging from 12.3-24.2\% (Table 4).

198

199

200

201

202

203

204

205

206

207

208

209

210

211

212

213

214

215

216

217

218

219

220

221

\section{Discussion}

For individual recognition to occur, animals must produce individually unique and stable cues, which their conspecifics will remember and use as a template for recognition during subsequent encounters. In this study, we demonstrate that wild male African savanna elephants produce individually distinct vocalizations that are stable over time and context and thus have the potential to be used for individual identification providing a basis for complex social associations to be established and maintained.

We showed that rumbles produced by male African savanna elephants were characteristic to a given animal and significantly different from that of other individuals. Vocalizations were distinct despite the animals being of the same sex and age category, and inhabiting the same area, pointing to true individual differences (differentiating each individual) rather than those resulting from other factors such as sex, age, or geographical region. All measured frequency parameters contributed relatively evenly to these differences, suggesting that it is the overall characteristics of the vocalizations rather than just one or several spectral parameters that encode identity. Pairwise comparisons further confirmed the robustness of individual differences in male vocalizations. The overall individual distinctiveness of acoustic cues was not driven by one or two individuals being very different from the rest but were a result of strong differences between a majority of elephants (Table 3), reflecting natural variation of vocalizations between individuals. While the large number of pairwise comparisons $(\mathrm{n}=10)$ and our conservative adjustment of $p$ values contributed to not all elephant dyads being significantly different in rumble acoustic parameters, it is also possible that this variation in dissimilarity correlates with relatedness (e.g. Charlton et al. 2009, Gamba et al. 2016) or social associations (e.g. Mitani and Brandt 1994) and warrants further investigation.

222 Previous research explored the distinctiveness of male African elephant rumbles in captivity 223 (Stoeger and Baotic 2016). The authors focused on age and size differences among males and 224 also showed that individuality can be encoded in rumbles. While providing important information about the call structure, the recordings were collected over a short period of time (average of 12 days per location) and thus the within-individual similarity could have potentially resulted from context- or state- dependent factors and the evaluation of the stability of the cues was not possible. Furthermore, the elephants were housed in four different institutions, and thus the observed differences among individuals could have been confounded by population or 
230 regional differences resulting from different origins or influence of associating conspecifics (as is

231 the case in some other mammals; Laimera et al. 2010). Our study allowed for testing wild 232 animals over a long time period (mean of 402.8 days between the first and last recording of the 233 same individual) to confirm the presence of individually distinct vocalizations while 234 concurrently indicating the robustness of male vocalizations over time. Rumbles are used by 235 African elephants in many different contexts (Moss et al. 2011) and the vocalizations used in our 236 analysis were recorded while elephants were displaying various behaviors. Despite this, the 237 individual differences were pronounced, suggesting that rumbles can provide reliable 238 information about identity across a variety of behavioral contexts.

239 Male-male interactions are often competitive as they are frequently related to resource 240 acquisition (van Hooff and van Schaik 1994). This is the case for elephants, where males 241 compete for females and resources, with high aggression rates occurring among adults (Lee et al. 242 2011), particularly during reproduction. In the context of these behaviors, acoustic cues 243 (allowing for the transmission of information over large distances; Bradbury and Vehrencamp 244 2011) combined with knowledge about the outcomes of previous encounters, the identification of 245 individuals through acoustic cues allows for the evaluation of risk at a distance, and an

246 adjustment of behavior, potentially limiting direct aggressive encounters and decreasing the risk 247 of injury. Because of this, studies of intra-sexual, male-male recognition have often focused on 248 competition and rival assessment (e.g. Casey et al. 2015, Charlton et al. Kitchen et al. 2003, 249 Pitcher et al. 2015, Reby et al. 2005). While this is a likely explanation for recognition to exist, 250 "true" (sensu Tibbetts and Dale 2007) individual recognition (defined as animals being able to 251 recognize multiple individuals, as opposed to distinguishing groups of individuals - e.g. 252 determined by age or sexual status) in context of intra-sexual interactions has been not studied 253 frequently (Carlson et al. 2020). Therefore, there remains ambiguity as to the recognition 254 abilities of many studied species. If variables that vary among individuals, such as body size or 255 256 257 258 259 age, are not adjusted for, then class-level recognition could be interpreted as individual recognition. By controlling for age and size in our study, we eliminated that possibility and found that cues were individually distinct, indicating that recognition in males could be used not only for rival assessment, but also for maintaining long-term affiliative associations during noncompetitive periods.

265 in a limited sample size as well as the impossibility of including formant frequencies (which may 266 also play an important role in animal communication) into the analyses. It is possible that these 267 factors could have contributed to the dissimilarity differences in our pairwise comparisons. It is 268 important that future studies investigate a wide variety of elephant rumble acoustic parameters to 
269

270

271

272

273

274

275

276

\section{7}

278

279

280

281

282

283

284

285

286

287

288

289

290

291

292

293

294

295

296

297

298

299

300

301

302

303

304

305

reveal the intricate characteristics of their acoustic signatures. Future research should also focus on experimentally confirming through bioassays whether acoustic cues are used by animals for individual recognition and if elephants only rely on certain features of the rumbles for recognition. Despite the limitations of our study, individual differences in the measured parameters (frequency and duration) were still evident, demonstrating that collecting acoustic samples from wild male elephants is possible and can provide useful data allowing for significant contributions to the study of animal communication.

\section{Conclusions}

We extend earlier studies of acoustic communication in elephants to investigate the structure and stability of social rumbles recorded from wild, free-ranging male elephants and evaluate their potential for conveying individual identity information. For individual recognition to occur, animals must not only produce individually distinct cues, but these cues must also be stable (Thom and Hurst 2004). We demonstrated that both these conditions were met and thus, an acoustic basis for individual recognition of male African elephants exists, is stable, robust and seems to be encoded in the overall rumble spectrum. Therefore, acoustic individual recognition is likely to occur in male African elephants. While mature male savannah elephants were previously considered to be primarily solitary, we now know that this is not the case (Chiyo et al. 2011; Goldenberg et al. 2014; Murphy et al. 2019). Instead, they exhibit a fission-fusion social structure, which sits against a backdrop of seasonally fluctuating resource availability and cyclic reproductive state. Adult male elephants in the studied population maintain some stability in social relationships over time (Murphy et al. 2019), however, these relationships are disrupted by musth (Goldenberg et al. 2014). Therefore, the ability to recognize long-term associates over time could be central to the stability of male elephant social strategies.

\section{Acknowledgements}

We thank Amy Morris Drake for beginning the collection of vocal samples under the guidance of HSM. We are grateful to Christin Winter, Jessica Wilmot and Tammy Eggeling from Elephants Alive for many hours of sound recordings. The Private landowners and Wardens of the Associate Private Nature Reserves are thanked for the permission to work on their land.

\section{References}

Anderson MJ. 2001. A new method for non-parametric multivariate analysis of variance. Austral Ecol. 26:32-46.

Baotic A, Stoeger AS. 2017. Sexual dimorphism in African elephant social rumbles. PLoS ONE $12: 1-14$. 
306 Black CE, Mumby HS, Henley MD. 2019. Mining morphometrics and age from past survey 307 photographs. Front Zool. 16:1-11.

308 Bradbury JW, Vehrencamp SL. 2011. Principles of animal communication. Sunderland, MA: 309 Sinauer Associates.

310 Caldwell MC, Caldwell DK. 1965. Individualized whistle contours in bottle-nosed dolphins 311 (Tursiops truncatus). Nature 207:434-435

312 Carlson NV, Kelly EMK, Couzin I. 2020. Individual vocal recognition across taxa: a review of 313 the literature and a look into the future. Philos. Trans. R. Soc. B, 375(1802), 20190479.

314 Casey C, Charrier I, Mathevon N, Reichmuth C. 2015. Rival assessment among northern 315 elephant seals: evidence of associative learning during male-male contests. R. Soc. open sci. 2, 316150228.

317 Charif R, Waack A, Strickman L. 2010. Raven Pro 1.4 User's Manual. Cornell Lab of 318 Ornithology, Ithaca, NY

319 Charlton BD, Whisson DA, Reby D. 2013. Free-ranging male koalas use size-related variation in 320 formant frequencies to assess rival males. PLoS One, 8(7):e70279.

321 Charlton BD, Zhihe Z., Snyder RJ. 2009. Vocal cues to identity and relatedness in giant pandas 322 (Ailuropoda melanoleuca). J. Acoust. Soc. Am., 126(5), 2721-2732.

323 Chiyo PI, Archie EA, Hollister-Smith JA, Lee PC, Poole JH, Moss CJ, Alberts SC. 2011. 324 Association patterns of African elephants in all-male groups: The role of age and genetic 325 relatedness. Anim Behav. 81:1093-1099.

326 Clarke KR. 1993. Non-parametric multivariate analyses of changes in community structure. 327 Aust. J. Ecol. 18:117-143.

328 Clemins PJ, Johnson MT, Leong KM, Savage A. 2005. Automatic classification and speaker 329 identification of African elephant (Loxodonta africana) vocalizations. J. Acoust. Soc. Am. 330 117:956-963.

331 Dabbs Jr JM, Mallinger A. 1999. High testosterone levels predict low voice pitch among men. 332 Pers Indiv Differ. 27(4):801-4.

333 Enquist M, PL Hurd, Ghirlanda S. 2010. Signalling. In: Westneat D, Fox C, editors. 334 Evolutionary behavioural ecology. Oxford, UK: Oxford University Press.

335 Falls JB. 1982. Individual recognition by sounds in birds. In: Kroodsma DE, Miller EH, editors. 336 Acoustic communication in birds. New York, NY: Academic Press. 
337 Fedurek P, Slocombe KE, Enigk DK, Thompson ME, Wrangham RW, Muller MN. 2016. The 338 relationship between testosterone and long-distance calling in wild male chimpanzees. Behav 339 Ecol Sociobiol. 70(5), 659-672.

340 Gamba M, Torti V, Bonadonna G, Randrianarison RM, Friard O, Giacoma C. 2016. Melody in 341 my head, melody in my genes? Acoustic similarity, individuality, and genetic relatedness in the 342 indris of Eastern Madagascar. J. Acoust. Soc. Am., 140(4), 3017-3018.

343 Ganswindt A, Rasmussen HB, Heistermann M, Hodges JK. 2005. The sexually active states of 344 free-ranging male African elephants (Loxodonta africana): defining musth and non-musth using 345 endocrinology, physical signals, and behavior. Horm Behav. 47: 83-91.

346 Goldenberg SZ, de Silva S, Rasmussen HB, Douglas-Hamilton I, Wittemyer G. 2014.

347 Controlling for behavioural state reveals social dynamics among male African elephants, 348 Loxodonta africana. Anim Behav. 95:111-119.

349 Hervé M. 2019. RVAideMemoire: Testing and Plotting Procedures for Biostatistics

350 Higham JP, Hebets EA. 2013. An introduction to multimodal communication. Behav Ecol 351 Sociobiol. 67:1381-1388.

352 Hollister-Smith JA, Alberts SC, Rasmussen LEL. 2008. Do male African elephants, Loxodonta 353 africana, signal musth via urine dribbling? Anim Behav. 76:1829-1841.

354 Kitchen DM, Seyfarth RM, Fischer J, Cheney DL. 2003. Loud calls as indicators of dominance 355 in male baboons (Papio cynocephalus ursinus). Behav. Ecol. Sociobiol. 53, 374-384.

356 Lee PC, Poole JH, Njiraini N, Sayialel S, Moss C. 2011. Male social dynamics: independence 357 and beyond. In: Moss CJ, Croze H, Lee PC, editors. The Amboseli Elephants: A long term 358 perspective on a long-lived mammal. Chicago, IL: University of Chicago Press. p. 260-271.

359 Leighty KA, Soltis J, Wesolek CM, Savage A. 2008. Rumble vocalizations mediate interpartner 360 distance in African elephants, Loxodonta africana. Anim Behav 76:1601-1608.

361 McComb K, Moss C, Sayialel S, Baker L. 2000. Unusually extensive networks of vocal 362 recognition in African elephants. Anim Behav. 59:1103-1109.

363 McComb K, Reby D, Baker L, Moss C, Sayialel S. 2003. Long-distance communication of 364 acoustic cues to social identity in African elephants. Anim Behav, 65:317-329.

365 Mitani JC, Brandt KL. 1994. Social factors influence the acoustic variability in the long-distance 366 calls of male chimpanzees. Ethology, 96:233-52. 
367 Moss CJ, Croze H, Lee PC. 2011. The Amboseli elephants: a long-term perspective on a long368 lived mammal. Chicago, IL: University of Chicago Press.

369 Morris-Drake A., Mumby HS. 2018. Social associations and vocal communication in wild and 370 captive male savannah elephants Loxodonta africana. Mamm Rev, 48:24-36.

371 Murphy D, Mumby HS, Henley MD. 2019. Age differences in the temporal stability of a male 372 African elephant (Loxodonta africana) social network. Behav Ecol. arz152.

373 Oksanen J, Blanchet FG, Friendly M, Kindt R, Legendre P, McGlinn D, Minchin PR, O'Hara 374 RB, Simpson GL, Solymos P, Henry M, Stevens H, Szoecs E, Wagner H. 2019. Vegan:

375 Community Ecology Package.

376 Owings, DH, Morton ES. 1998. Animal vocal communication: a new approach. Cambridge, UK: 377 Cambridge University Press.

378 Pasch B, George AS, Hamlin HJ, Guillette Jr LJ, Phelps SM. 2011. Androgens modulate song 379 effort and aggression in Neotropical singing mice. Horm Behav. 59(1):90-7.

380 Pitcher BJ, Briefer EF, McElligott AG. 2015. Intrasexual selection drives sensitivity to pitch, 381 formants and duration in the competitive calls of fallow bucks. BMC Evol Biol. 15(1):149.

382 Poole JH. 1987. Rutting Behavior in African Elephants: The Phenomenon of Musth. Behaviour 383 102:283-316.

384 Poole JH. 1999. Signals and assessment in African elephants: evidence from playback

385 experiments. Anim Behav, 58(1), 185-193.

386 Poole, JH. 2011. Behavioral contexts of elephant acoustic communication. In: Moss CJ, Croze 387 H, Lee PC, editors. The Amboseli Elephants: A long term perspective on a long-lived mammal. 388 Chicago, IL: University of Chicago Press. p. 125-161.

389 R Core Team. 2020. R: A language and environment for statistical computing. R Foundation for 390 Statistical Computing, Vienna, Austria. URL https:/www.R-project.org/.

391 Reby D, McComb K, Cargnelutti B, Darwin C, Fitch WT, Clutton-Brock T. 2005. Red deer stags 392 use formants as assessment cues during intrasexual agonistic interactions. Proc. R. Soc. B, 272, 393 941-947.

394 Schulte BA, Rasmussen LE. 1999. Musth, sexual selection, testosterone, and metabolites. In:

395 Advances in chemical signals in vertebrates, Boston, MA: Springer.

396 Soltis J, Leighty KA, Wesolek CM, Savage A. 2009. The Expression of Affect in African

397 Elephant (Loxodonta africana) Rumble Vocalizations. Int J Comp Psychol. 123:222-225.

Peer] reviewing PDF | (2020:08:52158:2:0:NEW 15 Dec 2020) 
398 Soltis J, Leong K, Savage A. 2005a. African elephant vocal communication I: antiphonal calling 399 behaviour among affiliated females. Anim Behav. 70:579-587.

400 Soltis J, Leong K, Savage A. 2005b. African elephant vocal communication II: Rumble variation 401 reflects the individual identity and emotional state of callers. Anim Behav. 70:589-599. Soltis J. 402 2010. Vocal communication in African elephants (Loxodonta africana). Zoo Biol. 29:192-209.

403 Stoeger AS, Baotic A. 2017. Male African elephants discriminate and prefer vocalizations of 404 unfamiliar females. Sci Rep. 7:46892.

405 Stoeger AS, Baotic A. 2016. Information content and acoustic structure of male African elephant 406 social rumbles. Sci Rep. 6:1-8.

407 Stoeger AS, Zeppelzauer M, Baotic A. 2014. Age-group estimation in free-ranging African 408 elephants based on acoustic cues of low-frequency rumbles. Bioacoustics. 23:231-246.

409 Thom MD, Hurst JL. 2004. Individual recognition by scent. Ann Zool Fenn. 41:765-787.

410 Tibbetts EA, Dale J. 2007. Individual recognition: it is good to be different. Trends Ecol Evol. $41122: 529-537$.

412 van Hooff JARAM, van Schaik CP. 1994. Male bonds: Afilliative relationships among 413 nonhuman primate males. Behaviour 130:309-337.

414 Wierucka K, Charrier I, Harcourt R, Pitcher BJ. 2018a. Visual cues do not enhance sea lion 415 pups' response to multimodal maternal cues. Sci Rep. 1-7.

416 Wierucka K, Pitcher BJ, Harcourt R, Charrier I. 2018b. Multimodal mother-offspring 417 recognition: the relative importance of sensory cues in a colonial mammal. Anim Behav. 418 146:135-142.

419 Wesolek CM, Soltis J, Leighty KA, Savage A. 2009. Infant African elephant rumble 420 vocalizations vary according to social interactions with adult females. Bioacoustics. 18:227-239.

421 Wood JD, Mccowan B, Langbauer Jr WR, Viljoen JJ, Hart LA., 2005. Classification of African 422 elephant Loxodonta africana rumbles using acoustic parameters and cluster analysis.

423 Bioacoustics. 15:143-161.

424 Yorzinski JL (2017) The cognitive basis of individual recognition. Curr Opin. in Behav. Sci. 425 16:53-57. 


\section{Figure 1}

Example of a non-musth, mature African elephant male social rumble, with measurements that were used for analysis indicated on the spectrogram. Hann window size of $600 \mathrm{~ms}$, with a hop size of $300 \mathrm{~ms}$ and an overlap of $50 \%$.

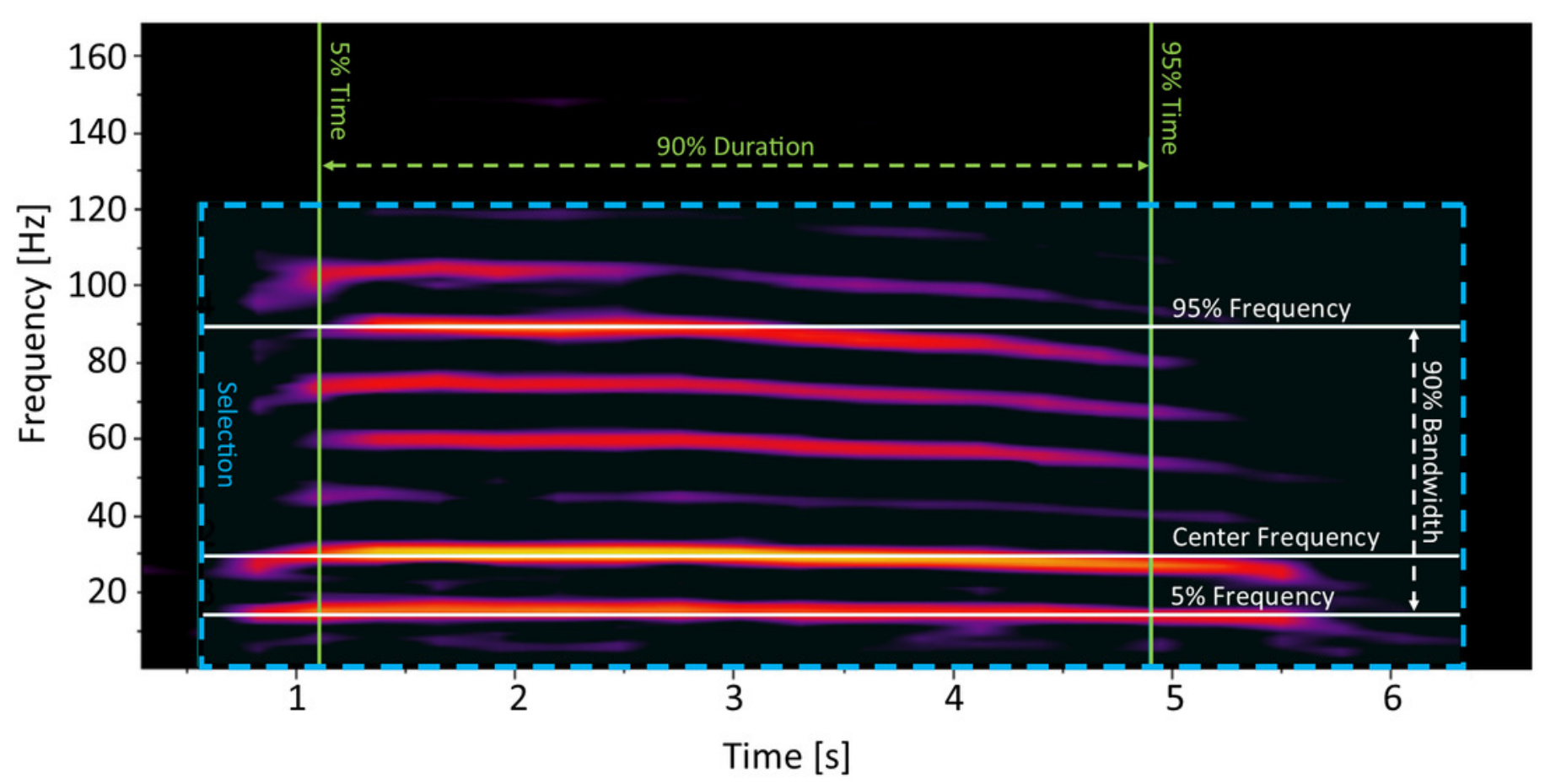




\section{Table $\mathbf{1}$ (on next page)}

Definitions of acoustic measurements collected for African elephant male rumbles (for accuracy, definitions are reproduced from Charif et al. 2010). 
Measurement

Definition

The frequency that divides the selection into two frequency intervals of

Center Frequency equal energy. It is the smallest discrete frequency in which the left side of the formula exceeds $50 \%$ of the total energy in the selection.

The frequency that divides the selection into two frequency intervals containing $5 \%$ and $95 \%$ of the energy in the selection. The computation

Frequency 5\% of this measurement is similar to that of Center Frequency, except that the summed energy has to exceed $5 \%$ of the total energy instead of $50 \%$.

The frequency that divides the selection into two frequency intervals containing $95 \%$ and $5 \%$ of the energy in the selection. The computation Frequency $95 \% \quad$ of this measurement is similar to that of Center Frequency, except that the summed energy has to exceed $95 \%$ of the total energy instead of $50 \%$.

Bandwidth 90\%

Bandwidth $90 \%$ is the difference between the $5 \%$ and $95 \%$ Frequencies.

The 5\% Time and 95\% Time are the points in time at which the selection is divided into two time intervals containing $5 \%$ and $95 \%$ or Duration $90 \%$ $95 \%$ and $5 \%$ of the energy in the selection, respectively. Therefore the $5 \%$ and $95 \%$ Time is the smallest discrete time in which the left side of the formula exceeds $5 / 95 \%$ of the total energy in the selection. Duration $90 \%$ is the difference between the $5 \%$ and $95 \%$ Times. 


\section{Table 2 (on next page)}

Sample size, range of dates, mean acoustic parameters ( \pm SE) of recorded vocalizations of each African savanna elephant male (A-E). 


\begin{tabular}{cccccc}
\hline & A & B & C & D & E \\
\hline First recording & $10 / 08 / 2016$ & $16 / 12 / 2016$ & $27 / 06 / 2016$ & $16 / 08 / 2016$ & $02 / 09 / 2016$ \\
Last recording & $04 / 10 / 2017$ & $04 / 10 / 2017$ & $12 / 09 / 2017$ & $26 / 10 / 2017$ & $26 / 10 / 2017$ \\
Median recording & $26 / 10 / 2016$ & $15 / 06 / 2017$ & $24 / 01 / 2017$ & $22 / 06 / 2017$ & $28 / 11 / 2016$ \\
Range of days & 421 & 293 & 443 & 437 & 420 \\
Sample size & 24 & 6 & 10 & 35 & 6 \\
\hline Duration 90\% $[\mathrm{s}]$ & $4.06( \pm 0.19)$ & $3.33( \pm 0.26)$ & $3.81( \pm 0.26)$ & $4.75( \pm 0.15)$ & $2.98( \pm 0.49)$ \\
Center Frequency $[\mathrm{Hz}]$ & $28.16( \pm 1.08)$ & $23.43( \pm 2.34)$ & $32.53( \pm 4.8)$ & $28.38( \pm 0.29)$ & $27.1( \pm 3.96)$ \\
Frequency 5\% $[\mathrm{Hz}]$ & $11.22( \pm 0.56)$ & $10.5( \pm 0.95)$ & $17.3( \pm 2.99)$ & $11.14( \pm 0.34)$ & $8.32( \pm 1.11)$ \\
Frequency 95\% $[\mathrm{Hz}]$ & $85.04( \pm 2.71)$ & $75.2( \pm 6.93)$ & $69.73( \pm 4.79)$ & $75.71( \pm 1.74)$ & $74.95( \pm 3.68)$ \\
Bandwidth [Hz] & $73.83( \pm 2.61)$ & $64.7( \pm 6.8)$ & $52.45( \pm 2.96)$ & $64.59( \pm 1.66)$ & $66.67( \pm 3.7)$ \\
\hline
\end{tabular}




\section{Table 3(on next page)}

Dissimilarity in African elephant male vocalizations.

Adjusted p-values from pairwise comparisons are shown. Significant results (in bold) indicate strong differences in vocalization parameters between individuals. Letters represent individuals (elephants). 


\begin{tabular}{c|cccc} 
B & 0.160 & & & \\
C & $\mathbf{0 . 0 0 1}$ & $\mathbf{0 . 0 1 7}$ & & \\
D & $\mathbf{0 . 0 0 3}$ & $\mathbf{0 . 0 0 1}$ & $\mathbf{0 . 0 0 1}$ & \\
E & 0.094 & 0.323 & $\mathbf{0 . 0 0 3}$ & $\mathbf{0 . 0 0 2}$ \\
\hline & A & B & C & D
\end{tabular}

1 


\section{Table 4 (on next page)}

The average contribution of each measured spectral parameter to the overall observed difference between African elephant male rumbles. 
Measured parameter

Duration 90\% [s]

Bandwidth $[\mathrm{Hz}]$

Frequency 95\% [Hz]

Center Frequency [Hz]

Frequency 5\% [Hz]
Average contribution [\%]

24.19

22.57

16.43

12.29

1 